\title{
Fast rotation and acoustic modes
}

\author{
Daniel Roy Reese
}

\author{
LESIA, Observatoire de Paris, Université PSL, CNRS, Sorbonne Université, Univ. Paris Diderot, \\ Sorbonne Paris Cité, 5 place Jules Janssen, 92195 Meudon, France
}

\begin{abstract}
In this contribution, we describe recent progress in modelling acoustic pulsations of rapidly rotating stars. We then go on to describe very recent attempts at interpreting pulsation spectra in two observed stars. These make use of multi-colour mode visibilities, mode excitation, and pulsation frequencies in order to constrain the identification of the observed modes.
\end{abstract}

Keywords: stellar pulsations - stellar rotation - stellar structure and evolution

\section{Introduction}

The majority of massive and intermediate mass stars rotate rapidly. Indeed, as shown in Royer (2009), a substantial proportion of these rotate with equatorial velocities faster than $100 \mathrm{~km} . \mathrm{s}^{-1}$. This induces a number of physical effects, namely centrifugal deformation, gravity darkening (e.g. Domiciano de Souza et al. 2003, Monnier et al. 2007), baroclinic flows including meridional circulation and differential rotation (e.g. Rieutord \& Espinosa Lara 2009), and a modified evolution (e.g. Meynet \& Maeder 2000). An extensive monograph on the effects of rotation on stars and their evolution was written a decade ago (Maeder 2009).

Much progress has been made in modelling these stars. For instance, the Geneva code (Eggenberger et al. 2008), STAREVOL (Palacios et al. 2003) and CESTAM (Marques et al. 2013) include prescriptions based on Zahn (1992) in order to include the effects of rotation on stellar evolution but approximate the centrifugal deformation. The Self-Consistent Field method (Jackson et al. 2005, MacGregor et al. 2007) and the Evolution STEllaire en Rotation code (Espinosa Lara \& Rieutord 2013, Rieutord et al. 2016) produce 2D static models which fully include centrifugal deformation, although the latter can mimic the effects of evolution by including a different composition for the core. In spite of this progress, many open questions remain. For instance, there are discrepancies in $\mathrm{Ni}$ enrichment in B-type stars from the Large Magellanic Cloud as a function of stellar rotation rate between observations and theory (Hunter et al. 2008, Brott et al. 2011) and it is not clear up to what rotation rate the Zahn (1992) prescription is valid. In order to address these open questions, it is necessary to look for observational constraints.

Some of the tightest observational constraints come from asteroseismology as this is currently the only way we have for probing internal stellar structure. However, two major hurdles need to be overcome before such a technique is applicable to rapidly rotating stars. The first is being able to accurate calculate pulsation modes in such stars. In the case of acoustic modes, centrifugal deformation plays a key role in mode frequencies and geometry (Reese et al. 2006, Lignières et al. 2009). 
Hence an accurate fully 2D pulsation code is needed. Such codes have only become available recently, e.g. TOP (Reese et al. 2006, 2009) and ACOR (Ouazzani et al. 2012). The second major hurdle is mode identification, i.e. finding the correspondence between theoretically calculated modes and observed pulsations (e.g. Goupil et al. 2005). Indeed, unlike solar-like stars, the pulsation frequencies in rapidly rotating stars are subject to complex patterns which are not easy to identify $(e . g$. Lignières et al. 2009). Furthermore, since these pulsations tend to be excited via the $\kappa$-mechanism, their amplitudes are subject to non-linear saturation and mode interactions, making them currently near to impossible to predict (e.g. Dziembowski et al. 1988). In these proceedings, we address both of these topics and provide examples of attempting to interpret observed pulsations. The following section deals with pulsation calculations.

\section{Pulsation calculations}

Before carrying out pulsation calculations, one needs an unperturbed model of a rotating star. In what follows we consider models from the following two codes:

- Self-Consistent Field (SCF) method (Jackson et al. 2005, MacGregor et al. 2007): this produces chemically homogeneous barotropic stellar models, i.e. lines of constant density, pressure and temperature coincide. The centrifugal deformation is fully taken into account. The energy conservation equation is only satisfied when averaged horizontally. As such these models are ideal for adiabatic pulsation calculations.

- Evolution STEllaire en Rotation (ESTER) code (Espinosa Lara \& Rieutord 2013, Rieutord et al. 2016): this produces models with a uniform or step-wise constant composition. Baroclinic effects are taken into account, i.e. lines of constant density, pressure and temperature do not coincide. This leads to a rotation profile which depends and depth and latitude and to meridional circulation. The energy conservation equation is satisfied locally. Accordingly, these models are ideal for both adiabatic and non-adiabatic calculations.

Once the equilibrium model has been obtained, we can calculate the pulsation modes by perturbing the the fluid dynamic equations around the equilibrium model:

$$
\begin{aligned}
0 & =\frac{\delta \rho}{\rho_{0}}+\vec{\nabla} \cdot \vec{\xi} \\
0 & =\Delta \Psi-4 \pi G\left(\rho_{0} \frac{\delta \rho}{\rho_{0}}-\vec{\xi} \cdot \vec{\nabla} \rho_{0}\right) \\
0 & =[\omega+m \Omega]^{2} \vec{\xi}-2 i \vec{\Omega} \times[\omega+m \Omega] \vec{\xi}-\vec{\Omega} \times(\vec{\Omega} \times \vec{\xi})-\vec{\xi} \cdot \vec{\nabla}\left(\varpi \Omega^{2} \vec{e}_{\varpi}\right)-\frac{P_{0}}{\rho_{0}} \vec{\nabla}\left(\frac{\delta P}{P_{0}}\right) \\
& +\frac{\vec{\nabla} P_{0}}{\rho_{0}}\left(\frac{\delta \rho}{\rho_{0}}-\frac{\delta P}{P_{0}}\right)-\vec{\nabla} \Psi+\vec{\nabla}\left(\frac{\vec{\xi} \cdot \vec{\nabla} P_{0}}{\rho_{0}}\right)+\frac{\left(\vec{\xi} \cdot \vec{\nabla} P_{0}\right) \vec{\nabla} \rho_{0}-\left(\vec{\xi} \cdot \vec{\nabla} \rho_{0}\right) \vec{\nabla} P_{0}}{\rho_{0}^{2}} \\
\frac{\delta P}{P_{0}} & =\Gamma_{1} \frac{\delta \rho}{\rho_{0}}
\end{aligned}
$$

where quantities with the subscript " 0 " are equilibrium quantities, those preceded by " $\delta$ " Lagrangian perturbations, and other quantities Eulerian perturbations. $\omega$ represents the pulsation frequency (as viewed from an inertial frame), $\rho$ the density, $\xi$ the Lagrangian displacement, $\Psi$ the gravitational potential, $G$ the gravitational constant, $m$ the azimuthal order, $\Omega$ the rotation profile, $P$ the pressure, and 
$\Gamma_{1}$ the first adiabatic exponent. The above set of equations make use of the adiabatic approximation, i.e. energy exchanges are neglected during the oscillations.

In order to take into account energy exchanges, i.e. to carry out non-adiabatic calculations, Eq. (4) needs to be replaced by the following equations:

$$
\begin{aligned}
i[\omega+m \Omega] \rho_{o} T_{o} \delta S & =\epsilon_{o} \rho_{o}\left(\frac{\delta \epsilon}{\epsilon_{o}}+\frac{\delta \rho}{\rho_{o}}\right)-\vec{\nabla} \cdot \delta \vec{F}+\vec{\xi} \cdot \vec{\nabla}\left(\vec{\nabla} \cdot \vec{F}_{o}\right)-\vec{\nabla} \cdot\left[(\vec{\xi} \cdot \vec{\nabla}) \vec{F}_{o}\right] \\
\delta \vec{F}^{\mathrm{R}} & =\left[\left(1+\chi_{T}\right) \frac{\delta T}{T_{o}}+\chi_{\rho} \frac{\delta \rho}{\rho_{o}}\right] \vec{F}_{o}^{\mathrm{R}} \\
& -\chi_{o}\left[T_{o} \vec{\nabla}\left(\frac{\delta T}{T_{o}}\right)+\vec{\xi} \cdot \vec{\nabla}\left(\vec{\nabla} T_{o}\right)-\vec{\nabla}\left(\vec{\xi} \cdot \vec{\nabla} T_{o}\right)\right]
\end{aligned}
$$

where $T$ is the temperature, $S$ the entropy, $\vec{F}$ the energy flux, $\vec{F}^{R}$ the radiative energy flux, $\chi$ is deduced from the opacity, and $\chi_{T}$ and $\chi_{\rho}$ its logarithmic derivatives with respect to temperature and density. To these must be added perturbed versions of the equations of state and opacities (e.g. Dupret 2002).

These equations, along with suitable boundary conditions, are then explicitly written out using a spheroidal coordinate system which follows the shape of the star before being discretised using spherical harmonics in the horizontal direction and finite differences or Chebyshev polynomials in the radial direction. The pulsation frequencies and modes are then obtained numerically using an eigenvalue solver. The typical relative accuracy on the frequencies, obtained with the Two-dimensional Oscillation Program (TOP, Reese et al. 2006, 2009), is $10^{-4}$ for a tabulated equation of state (EOS) and $10^{-7}$ to $10^{-8}$ for an analytical EOS. The relative accuracy of the excitation/damping rates when carrying out non-adiabatic calculations is typically $10^{-1}$ to $10^{-2}$, given its relatively small magnitude compared to the pulsation frequencies.

\section{Mode classification}

As in non-rotating stars, pulsation modes in rapidly rotating stars are also subdivided into various categories. At low pulsation frequencies, the restoring force is a combination buoyancy and the Coriolis force, thus leading to gravito-inertial modes. These modes are described in Ouazzani (these proceedings). At high frequencies, the restoring force is pressure, hence the modes are acoustic modes. These are strongly affected by the centrifugal deformation and accordingly are subdivided into different classes at sufficient rotation rates, as shown by Lignières \& Georgeot $(2008,2009)$ using ray dynamics. These classes are characterised by different geometries and frequency patterns. They include 2-period island modes which are focused around a 2-periodic ray orbit which circumvents the equator, 6-period island modes, whispering gallery modes which resemble their non-rotating counterparts, and chaotic modes for which the nodes are irregularly spaced. Three of these classes are illustrated in Fig. 1.

Of these different classes, (2-period) island modes are the most interesting since these are the most visible of the regular (i.e. non-chaotic) modes. They are characterised by new quantum numbers as illustrated in the left panel of Fig. 1. Their frequencies obey the following asymptotic formula, as shown empirically in Reese et al. (2009):

$$
\omega \simeq \Delta_{\tilde{n}} \tilde{n}+\Delta_{\tilde{\ell}} \tilde{\ell}+\frac{\Delta_{\tilde{m}} m^{2}}{\tilde{n}}-m \Omega+\tilde{\alpha}
$$

The parameter $\Delta_{\tilde{n}}$ is the inverse of twice the travel time of an acoustic wave along the orbit (Lignières $\&$ Georgeot 2008, 2009). It was also shown to scale with the mean density, both theoretically (Reese 

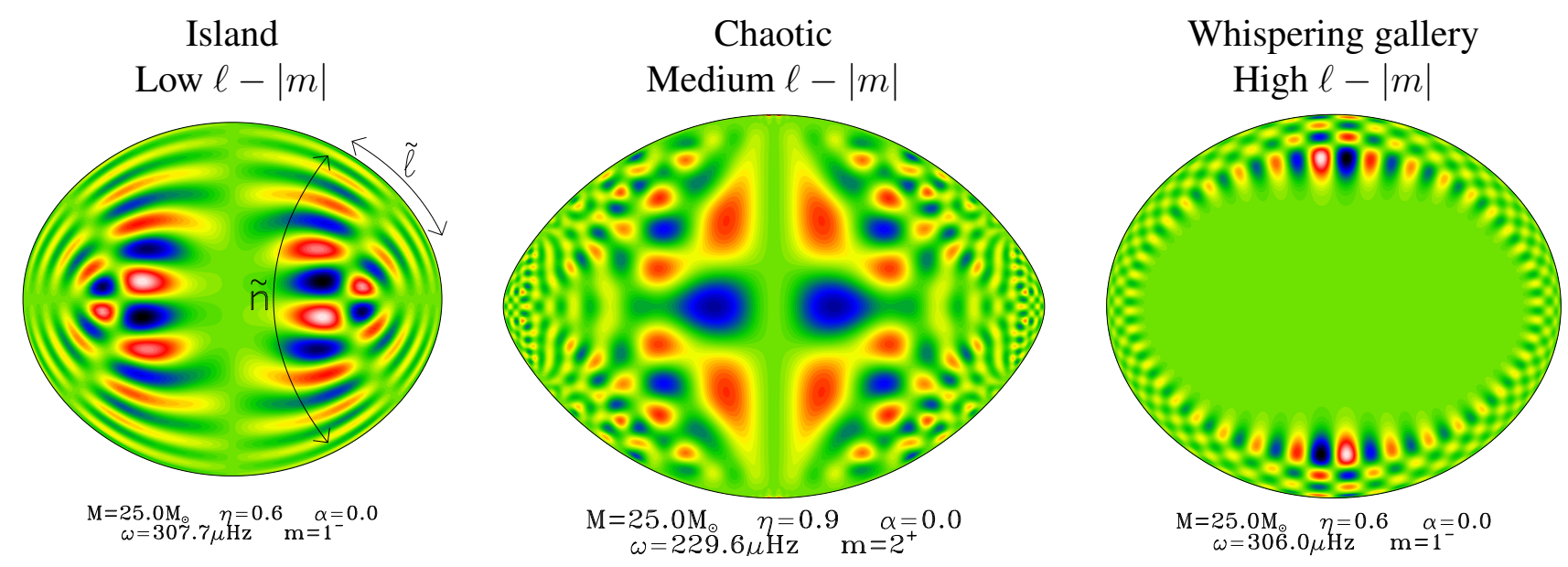

Figure 1: Meridional cross-sections of acoustic modes from different classes (taken from Reese et al. 2009).

et al. 2008) and observationally, using stars in binary systems (García Hernández et al. 2015). A semianalytical formula has also been derived for $\Delta_{\tilde{\ell}}$ (Pasek et al. 2011, 2012). When there is differential rotation, the parameter $\Omega$ is replaced by an average value of the rotation rate (Reese et al. 2009). A more precise formulation shows that the generalised rotational splitting, i.e. the frequency difference between a prograde mode and its retrograde counterpart, is related to the underlying rotation profile via the following integral formula (Reese et al. in prep.):

$$
\frac{\omega_{-m}-\omega_{m}}{2 m} \simeq \frac{\Omega_{\mathrm{m}}^{\mathrm{eff}}+\Omega_{-\mathrm{m}}^{\mathrm{eff}}}{2}+\frac{\mathcal{C}_{\mathrm{m}}+\mathcal{C}_{-\mathrm{m}}}{2}
$$

where

$$
\Omega_{\mathrm{eff}}=\frac{\int_{V} \Omega \rho_{o}\|\vec{\xi}\|^{2} d V}{\int_{V} \rho_{o}\|\vec{\xi}\|^{2} d V} \quad \mathcal{C}=\frac{i}{m} \frac{\int_{V} \rho_{o} \vec{\Omega} \cdot\left(\overrightarrow{\xi^{*}} \times \vec{\xi}\right) d V}{\int_{V} \rho_{o}\|\vec{\xi}\|^{2} d V}
$$

We note that here, we are using the retrograde convention, i.e. positive values of $m$ correspond to retrograde modes. The left panel of Fig. (2) compares the generalised rotational splittings with the right-hand side of Eq. (8) whereas the right panel shows a rotation kernel for an island mode, based on Eq. (9).

\section{Interpreting observations}

Having described the theory behind stellar oscillations in rapidly rotating stars as well as some of the effects of rotation on acoustic modes and their classification, we now turn our attention to observed stars. In particular, we will look at two recently studied stars and show how theoretical developments are providing new ways to interpret observations.

\section{1 $\alpha$ Ophiuchi}

The star $\alpha$ Ophiuchi (a.k.a. Rasalhague) is in fact a binary system composed of an A5III primary component and a K6V secondary star (Cowley et al. 1969, Hinkley et al. 2011). The primary component rotates with an equatorial velocity of $240 \mathrm{~km} \cdot \mathrm{s}^{-1}$ and its polar and equatorial radii have 

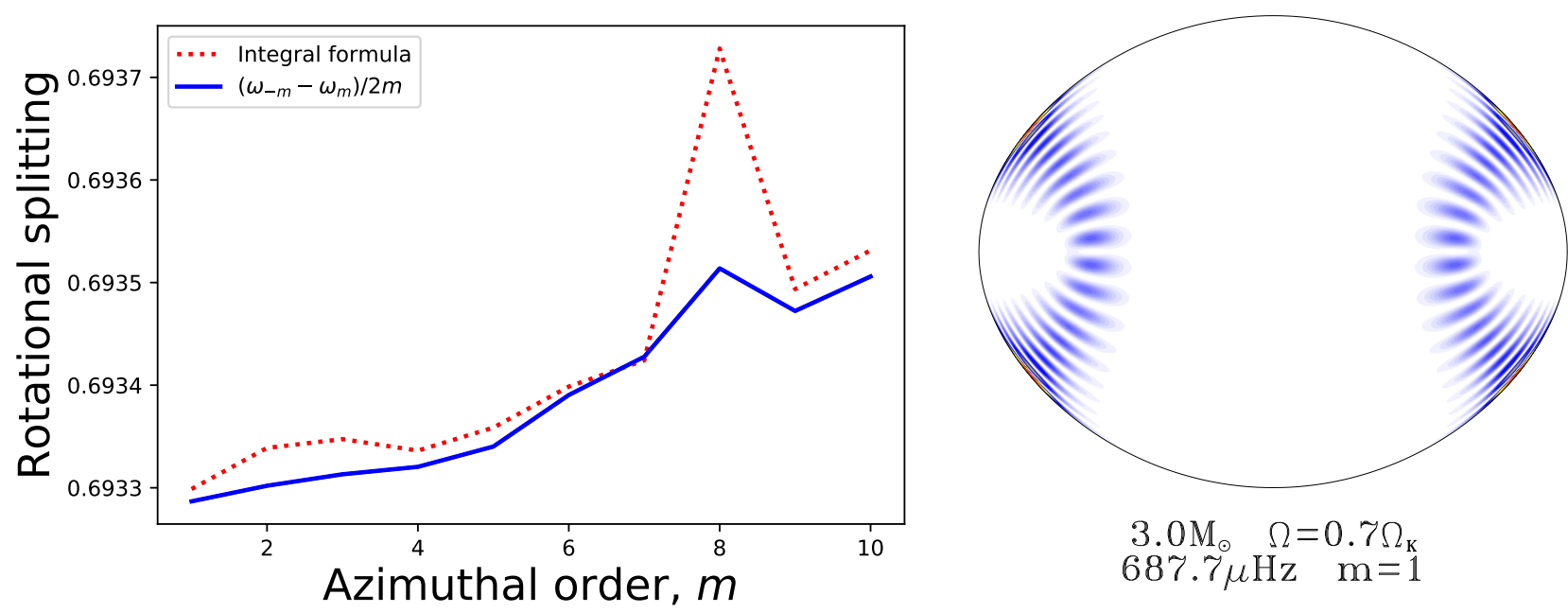

Figure 2: (Left): Generalised splittings vs. integral formula. (Right): rotation kernel for an island mode (from Reese et al. in prep.).

been determined through interferometry (Zhao et al. 2009). It pulsates with at least 57 pulsation modes as shown through MOST photometry (Monnier et al. 2010).

Mirouh et al. (2017) recently studied this star in an attempt to identify and interpret the stellar pulsations. Using the ESTER code, we first calculated a model that reproduces the luminosity, and the polar and equatorial radii. The parameters of the model are: $M=2.22 M_{\odot}, Z=0.02, X=0.7$, and $X_{c}=0.26$ (where $X_{c}$ is the central hydrogen abundance). We then went on to carry out nonadiabatic pulsation calculations with the TOP code. In order to increase the numerical stability of the calculations, it was necessary to use a hybrid approach where the adiabatic equations are used in the internal part of the star and the full non-adiabatic equation applied only in the external part where non-adiabatic effects are non-negligible. We calculated the mode visibilities (i.e. disk-integration geometric factors) using the formula:

$$
\delta \mathcal{I}=\iint_{\text {Vis.Surf. }} \delta I\left(g_{\text {eff }}, T_{\text {eff }}, \mu\right) \vec{e}_{\text {obs. }} \cdot \overrightarrow{d S}+\iint_{\text {Vis.Surf. }} I\left(g_{\text {eff }}, T_{\text {eff }}, \mu\right) \vec{e}_{\text {obs. }} \cdot \delta(\overrightarrow{d S})
$$

where $I$ is the specific intensity at each point on the stellar surface, and $\overrightarrow{d S}$ a surface element. We then looked for the modes that are the most visible and those that are excited as a potential match to the observations. Unfortunately, the only excited modes were high-degree gravity modes which are unlikely to correspond to the observed pulsations. The lack of excited acoustic modes is likely due to limitations in the ESTER code (which currently doesn't model outer convection zones) and/or the TOP pulsation code.

Even in spite of the inconclusive results, this study is an important first step in interpreting the observations. Indeed, it combines several independent constraints namely mode frequencies, visibilities and non-adiabatic mode excitation in order to constrain the mode identification. Future studies will also need to adopt a similar multi-faceted strategy in order to interpret the observations given the complexity of the pulsation spectra.

\section{2 $\beta$ Pictoris}

$\beta$ Pic is an A6V type star rotating with an equatorial projected velocity of $v \cdot \sin i=124 \pm 3 \mathrm{~km} . \mathrm{s}^{-1}$ (Koen 2003). Interferometric observations of a disk and an exoplanet orbiting around this star has 
sparked considerable interest thus leading to observations from multiple ground and space-based instruments, especially at the time of the expected transit of the exoplanet's Hill sphere. Consequently, 15 pulsation modes have been observed in up to 5 photometric passbands (Zwintz et al., subm., and references therein).

Using multicolour photometry is a particularly promising way of identifying pulsation modes in rapid rotators (e.g. Daszynska-Daszkiewicz et al. 2013, Reese et al. 2017). Indeed, as explained in the introduction, the intrinsic mode amplitudes of classical pulsators are near to impossible to predict. However, when calculating the ratios of mode amplitudes between different photometric bands, the intrinsic amplitude factors out, thus leaving a ratio of mode visibilities which can be calculated via Eq. (10) (in the relevant bands). Hence, Zwintz et al. (subm.) applied this strategy when interpreting $\beta$ Pictoris' pulsations.

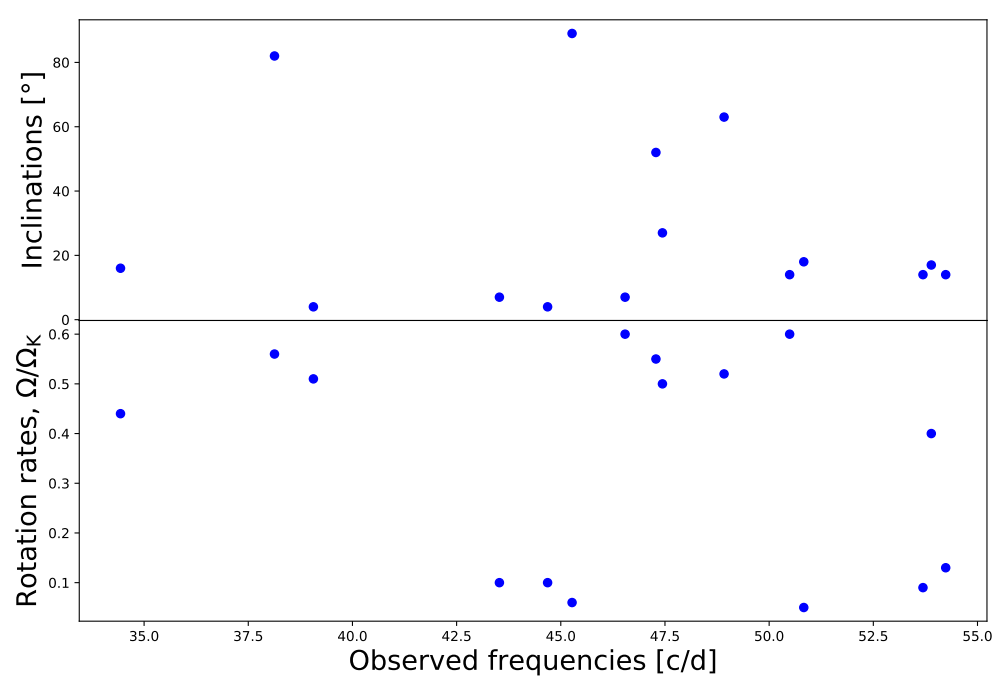

Figure 3: Inclinations and rotation rates obtained by fitting the multi-colour amplitudes of the pulsation modes of $\beta$ Pic individually (taken from ?).

As a first step, the multi-colour pulsation amplitudes of the individual modes were fit using theoretical pseudo non-adiabatic mode visibilities using rotating $1.8 M_{\odot} \mathrm{SCF}$ models. Although the amplitudes are well reproduced in most cases, the rotation rates and inclinations obtained for the different modes do not converge to a common value as illustrated in Fig. 3. This highlighted the need for imposing a common rotation rate and inclination and carrying out a global fit of all of the amplitudes and pulsation frequencies simultaneously. Such fits were therefore carried out using an MCMC strategy where the rotation rate, the inclination, and a scale factor on the frequencies were treated as free parameters. Furthermore, the amount of difference between the observed and theoretical frequencies was treated as a adjustable parameter thus allowing us to either enforce a tight fight to the pulsation spectrum, or a closer fit to the mode amplitudes. This lead to a variety of different solutions such as the one illustrated in Fig. 4. This particular solution was obtained with a relatively high tolerance on the frequency error and corresponds to a model with the parameters: $\Omega=0.27 \Omega_{\mathrm{K}}$, $i=89.9^{\circ}, R=1.66 R_{\odot}$ and $M=1.80 M_{\odot}$. Given the near equator-on inclination of the planetary orbit and debris disk, a high inclination of the star also seems likely. Nonetheless, large differences remain between the theoretical predictions and observations thus pointing to limitations in the model and raising the question as to whether the set of modes used in the interpretation, namely $\ell=0$ to 3 
modes, is too restrictive.
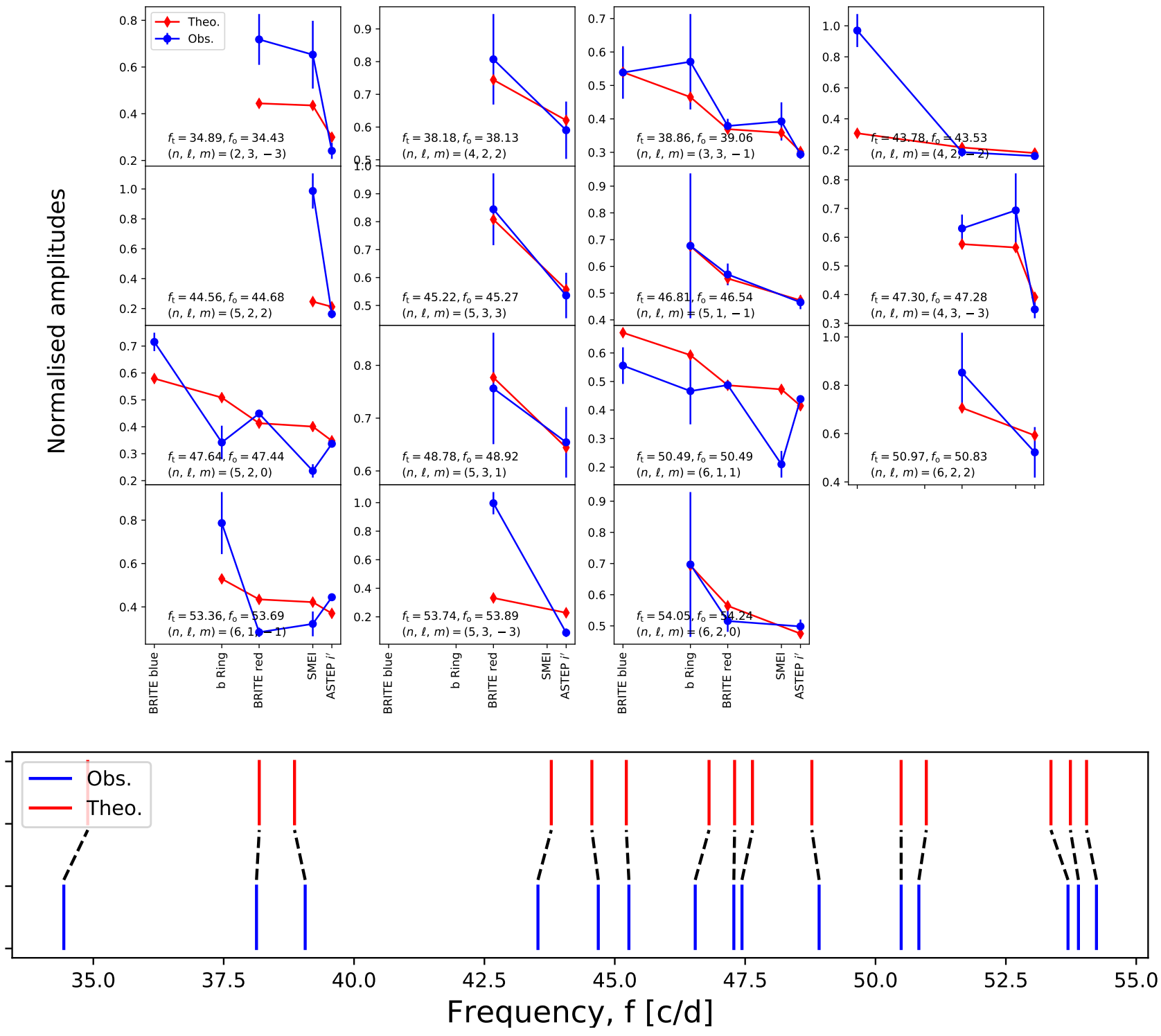

Figure 4: One of the solutions when carrying out a global fit of the multi-colour pulsation amplitudes (upper panel) and frequency spectrum (lower panel) of $\beta$ Pictoris (taken from ?).

\section{Conclusion}

As can be seen, much progress has been made in modelling pulsations of rapidly rotating stars. Indeed, the pulsation calculations are becoming more and more realistic with the inclusion of supplementary ingredients such as non-adiabatic effects. It is also possible to predict multi-colour mode visibilities as well as line profile variations. Frequency patters are starting to appear in pulsation spectra of such stars, even if they still remain difficult to interpret, and various attempts have been made at identifying modes. 
The next steps include extending mode identification techniques by developing more realistic fully non-adiabatic calculations, using line profile variations, and interpreting other pulsating rotating stars. Also, applying grid modelling techniques should help with deciphering the complex frequency patterns present in pulsation spectra of such stars. In this regard, the automatic mode classification recently set up by Mirouh et al. (2019) should greatly help with the production of theoretical spectra. A systematic study of non-adiabatic effects will reveal which modes are excited as a function of rotation rate. If a sufficient number of modes are identified using a combination of the above constraints, then it may be possible to probe the internal structure and rotation profile of rapidly rotating stars thus providing precious information on internal physical phenomena such as transport processes that take place in these stars.

\section{Acknowledgements}

DRR acknowledges the support of the "Programme National de Physique Stellaire" (PNPS) of CNRS/INSU co-funded by CEA and CNES as well as of the support of the French "Agence Nationale de la Recherche" to the ESRR project under grant ANR-16-CE31-0007.

\section{References}

Brott I., Evans C. J., Hunter I., et al. 2011, A\&A, 530, A116

Cowley A., Cowley C., Jaschek M., \& Jaschek C. 1969, Astr. J., 74, 375

Daszyńska-Daszkiewicz J., Szewczuk W., \& Walczak P. 2013, MNRAS, 431, 3396

Domiciano de Souza A., Kervella P., Jankov S., et al. 2003, A\&A, 407, L47

Dupret M.-A. 2002, PhD thesis, Université de Liège

Dziembowski W., Krolikowska M., \& Kosovichev A. 1988, AcA, 38, 61

Eggenberger P., Meynet G., Maeder A., et al. 2008, Ap\&SS, 316, 43

Espinosa Lara F. \& Rieutord M. 2013, A\&A, 552, A35

García Hernández A., Martín-Ruiz S., Monteiro M. J. P. F. G., et al. 2015, ApL, 811, L29

Goupil M.-J., Dupret M. A., Samadi R., et al. 2005, JA\&A, 26, 249

Hinkley S., Monnier J. D., Oppenheimer B. R., et al. 2011, ApJ, 726, 104

Hunter I., Brott I., Lennon D. J., et al. 2008, ApJ, 676, L29

Jackson S., MacGregor K. B., \& Skumanich A. 2005, ApJS, 156, 245

Koen C. 2003, MNRAS, 341, 1385

Lignières F. \& Georgeot B. 2008, Phys. Rev. E, 78, 016215

Lignières F. \& Georgeot B. 2009, A\&A, 500, 1173

MacGregor K. B., Jackson S., Skumanich A., \& Metcalfe T. S. 2007, ApJ, 663, 560

Maeder A. 2009, Physics, Formation and Evolution of Rotating Stars Astronomy and Astrophysics Library (SpringerVerlag)

Marques J. P., Goupil M. J., Lebreton Y., et al. 2013, A\&A, 549, A74

Meynet G. \& Maeder A. 2000, A\&A, 361, 101

Mirouh G. M., Angelou G. C., Reese D. R., \& Costa G. 2019, MNRAS, 483, L28

Mirouh G. M., Reese D. R., Rieutord M., \& Ballot J. 2017, in SF2A-2017: Proceedings of the Annual meeting of the French Society of Astronomy and Astrophysics, ed. C. Reylé P. Di Matteo F. Herpin E. Lagadec A. Lançon Z. Meliani \& F. Royer 103-106

Monnier J. D., Townsend R. H. D., Che X., et al. 2010, ApJ, 725, 1192

Monnier J. D., Zhao M., Pedretti E., et al. 2007, Science, 317, 342

Ouazzani R.-M., Dupret M.-A., \& Reese D. R. 2012, A\&A, 547, A75

Palacios A., Talon S., Charbonnel C., \& Forestini M. 2003, A\&A, 399, 603

Pasek M., Georgeot B., Lignières F., \& Reese D. R. 2011, Physical Review Letters, 107, 121101

Pasek M., Lignières F., Georgeot B., \& Reese D. R. 2012, A\&A, 546, A11

Reese D., Lignières F., \& Rieutord M. 2006, A\&A, 455, 621

Reese D., Lignières F., \& Rieutord M. 2008, A\&A, 481, 449 
Reese D. R., Lignières F., Ballot J., et al. 2017, A\&A, 601, A130

Reese D. R., MacGregor K. B., Jackson S., Skumanich A., \& Metcalfe T. S. 2009, A\&A, 506, 189

Rieutord M. \& Espinosa Lara F. 2009, Communications in Asteroseismology, 158, 99

Rieutord M., Espinosa Lara F., \& Putigny B. 2016, Journal of Computational Physics, 318, 277

Royer F. 2009, in Lecture Notes in Physics, Berlin Springer Verlag, Vol. 765, The Rotation of Sun and Stars, ed. J.-P. Rozelot \& C. Neiner 207-230

Zahn J.-P. 1992, A\&A, 265, 115

Zhao M., Monnier J. D., Pedretti E., et al. 2009, ApJ, 701, 209

Zwintz K., Reese D. R., Neiner C., \& et a. 2019, A\&A, submitted 